\title{
Comprehensive line-spread function error budget for the off-plane grating rocket experiment (Erratum)
}

\author{
Benjamin D. Donovan, ${ }^{\mathrm{a}, *}$ Randall L. McEntaffer, ${ }^{\mathrm{a}}$ James H. Tutt, ${ }^{\mathrm{a}}$ \\ Bridget C. O'Meara, ${ }^{a}$ Fabien Grisé, ${ }^{\text {a }}$ William W. Zhang, \\ Michael P. Biskach, ${ }^{b}$ Timo T. Saha, ${ }^{b}$ Andrew D. Holland, \\ Daniel Evan, ${ }^{c}$ Matthew R. Lewis, ${ }^{c}$ Matthew R. Soman, ${ }^{c}$ \\ Karen Holland, ${ }^{d}$ David Colebrook, ${ }^{d}$ Fraser Cooper, ${ }^{d}$ and \\ David Farn ${ }^{\text {d }}$ \\ ${ }^{a}$ The Pennsylvania State University, University Park, Pennsylvania, United States \\ bNASA Goddard Space Flight Center, Greenbelt, Maryland, United States \\ ${ }^{c}$ The Open University, Milton Keynes, United Kingdom \\ ${ }^{\mathrm{d} X C A M}$ Ltd., Northampton, United Kingdom
}

[DOI: 10.1117/1.JATIS.7.1.019801]

This article [J. Astron. Telesc. Instrum. Syst. 7(1), 01400321 January 2021 https://doi.org/10 .1117/1.JATIS.7.1.014003] was originally published with a value misaligned in Table 2. The value "21.8" appeared under the "arcsec" column in the "Grat. res. limit" row. The value should have appeared under the "Disp." column in the same row.

The article was corrected on 27 January 2021.

*Address all correspondence to Benjamin D. Donovan, bdonovan@psu.edu 